\title{
The Eigenfunction Method for Determining Displacement Time History in Structural Dynamic Analysis
}

\author{
Bo Li', Mahesh D. Pandey², Yang Lu', Kao-Shan Dai'* \\ ${ }^{1}$ Key Laboratory of Deep Underground Science and Engineering (Ministry of Education), Department of Civil Engineering, \\ College of Architecture and Environment, Sichuan University, Jiuyanqiao Wangjiang Road, Chengdu, 610064, China \\ 2 Department of Civil and Environmental Engineering, University of Waterloo, University Avenue West, Waterloo, ON, Canada \\ * Corresponding author, e-mail: kdai@scu.edu.cn
}

Received: 09 April 2019, Accepted: 03 October 2019, Published online: 30 October 2019

\begin{abstract}
In condition monitoring of structures, acceleration time histories are usually recorded due to ease of instrumentation. In cases where the information about a displacement time history is required, the acceleration data needs to be integrated to obtain the velocity and then the velocity needs to be integrated to obtain the displacements. However, the numerical integration of the acceleration data usually introduces an unrealistic drift component to the velocity as well as displacement. This paper presents an eigenfunction method to derive velocity and displacement time histories from a given acceleration time history. The paper analyzes displacements in two case studies using the numerical integration as well as the proposed eigenfunction method. It is concluded that the eigenfunction method is a viable approach to derive the displacement information from the acceleration data.
\end{abstract}

Keywords

eigenfunction method, drift, displacement, structural dynamic analysis

\section{Introduction}

The displacement time history of structures under dynamic effects is useful in (1) detecting effects of soil-structure interaction [1], (2) determining forces acting on a soil-structure interface in the substructure method [2], and (3) determining inter-story drift ratios of buildings under seismic excitations in performance-based seismic design $[3,4]$. However, in practice, it is extremely difficult and cumbersome to measure displacement time histories buildings.

Because the acceleration time history is easily measured by accelerometers, numerical integration is usually used to determine velocity and displacement from the measured acceleration. However, numerical integration often leads to unrealistic and large drifts in the velocity and displacement $[2,5]$. Reasons for the drifts in velocity and displacement have been studied for a long time [6, 7]. Previous studies showed that an offset in acceleration records caused by the instrument could lead to the drift. In addition, the random noise in acceleration records could also cause the drift in velocity and displacement during single or double integration.
To resolve the problem of drift, the method of baseline correction and the method of filtering have been developed in previous studies $[2,8,9]$ to adjust the original time histories. The baseline correction method applies some forms of polynomials to correct the original acceleration, and thus, obtain corrected acceleration without drift. However, Iwan and Chen [10] showed that velocity and displacement may be underestimated due to the baseline correction. Boore [6] also pointed out that baseline correction would remarkably change displacement response spectrum of the original ground motions at long periods. In addition, the baseline correction may still yield unreasonable displacements (i.e., remarkable drift), which was demonstrated by laboratory shaking table tests on digital instruments $[2,11]$. The acausal Fourier filtering is another method to eliminate the drift in displacement time history [9]. This method needs the original acceleration time history to be padded with zeros at both ends, and thus adds considerable length. This method requires the zero-padded portion to obtain accurate velocity and displacement waveforms when integrating from zero. Consequently, it may need more analysis runtime for structural dynamic analysis [12]. 
Li et al. [5] investigated reasons causing the drift in velo-city and displacement time histories by integrating acceleration. Their study reported that, in addition to conventionally recognized reasons (such as offset and random noise in acceleration records) causing the drift in velocity and displacement $[6,8]$, the over-determinacy associated with integration is another reason contributing to such drift. To overcome the over-determinacy, Li et al. [5] further developed eigenfunctions associated with a sixth-order differential equation, which satisfies all necessary boundary conditions of the problem.

Since the eigenfunction was developed by Li et al. [5], different methods have been proposed to modify real recorded ground motion using the eigenfunction to generate drift-free spectrum-matched ground motions $[5,13]$. However, the capability of eigenfunction to determine displacement time history in structural dynamic analysis remains unknown.

Based on previous studies regarding reasons contributing to the drift in displacement time histories, the objective of this study is to propose the eigenfunction method to determine velocity and displacement time histories from acceleration data, resolving the drift in time histories caused by the over-determinacy. The paper is organized as follows. Section 2 introduces the eigenfunction method that involves improvement of the acceleration integration over the study of $\mathrm{Li}$ et al. [5]. The Section 3 validates the eigenfunction method to determine velocity and displacement. Then, the Section 4 presents two examples that use the eigenfunction method to determine velocity and displacement time histories of vibrating bridges. From these two examples, capabilities of the eigenfunction method to determine displacement and velocity from acceleration data are illustrated. Conclusions of this study are summarized in the last section of this paper.

It is noted that, the eigenfunction method determines displacement time history under the assumption that displacement time history that exactly or approximately satisfies zero initial and end conditions; discussion of determining displacement time history for structures presenting damage and nonlinear response is beyond the scope of this current work. Also, pre-processing steps, such as removing noise and dealing with distortions and errors from various sources containing in the measured acceleration, are still required before the application of the eigenfunction method. The eigenfunction method focuses on eliminating the drift in displacement caused by over-determinacy associated with integration.

\section{The eigenfunction method}

\subsection{Drift in displacement and velocity}

Velocity $V(t)=\dot{u}(t)$ is the integration of acceleration $A(t)=\ddot{u}(t)$, and displacement $D(t)=u(t)$ is the integration of velocity $V(t)=\dot{u}(t)$. If $A(t), V(t)$, and $D(t)$ satisfy these relationships, they are called consistent time histories in this study. If structures do not undergo any permanent deformation under dynamic effects, their acceleration, velocity, and displacement responses should satisfy the zero initial and end conditions, i.e.,

$$
A(0)=A(T)=V(0)=V(T)=D(0)=D(T)=0,
$$

where $T$ is the duration of the time histories.

A realistic, non-drifting displacement process, which is consistent with the accelerogram associated, is paramount in dynamic analysis of structures, such as multiple support excitation analysis of large-span space structures. Some supports may be on different parts of a structure, and each support point may undergo different displacements. Multiple support excitation analysis of large-span space structures needs to consider seismic anchor movements and the inertia effects. If the calculated displacement presents drift or the baselines are improperly corrected, the analysis results from the incorrect displacement inputs may lead to an erroneous conclusion about the design [14].

In addition to conventional reasons (such as an offset in acceleration records, and random noise) causing the drift in velocity and displacement, it is important to understand the over-determinacy associated with integration contributing to such drift. To illustrate the contribution, consider an acceleration time history:

$$
A(t)=\sum_{k=1}^{n} \varepsilon_{k} \cdot(\sin ) \frac{(2 k \pm 1) \pi t}{T},
$$

where $n$ denotes the number of terms for the sine functions, and $\varepsilon_{k}$ is a small positive constant. Obviously, acceleration $A(t)$ given by Eq. (2) satisfies the zero initial and end conditions. Integrating Eq. (2) yields the velocity and displacement:

$$
\begin{aligned}
& A(t)=\sum_{k=1}^{n} \varepsilon_{k} \cdot(\sin ) \frac{(2 k \pm 1) \pi t}{T}, \\
& D(t)=-\sum_{k=1}^{n} \varepsilon_{k} \cdot\left[\frac{T}{(2 k \pm 1) \pi}\right]^{2} \sin \frac{(2 k \pm 1) \pi t}{T}+C_{1} t+C_{0} .
\end{aligned}
$$

In Eqs. (3) and (4), $V(t)$ and $D(t)$ must satisfy the last four zero initial and end conditions in Eq. (1). However, there are only two constants, i.e., $C_{0}$ and $C_{1}$, in the equations. As 
a result, the problem becomes over-determinate. Suppose that $V(t)$ and $D(t)$ are made to satisfy the zero initial and end conditions:

1. From $V(0)=0$, Eq. (3) gives $C_{1}=\sum_{k=1}^{n} \varepsilon_{k} \cdot \frac{T}{(2 k+1) \pi}$.

2. From $D(0)=0$, Eq. (4) gives $C_{0}=0$. Hence,

$$
\begin{aligned}
& V(t)=\sum_{k=1}^{n} \varepsilon_{k} \cdot \frac{T}{(2 k+1) \pi}\left[1-\cos \frac{(2 k+1) \pi t}{T}\right], \\
& D(t)=-\sum_{k=1}^{n} \varepsilon_{k} \cdot\left[\frac{T}{(2 k+1) \pi}\right]^{2} \sin \frac{(2 k+1) \pi t}{T} \\
& +\sum_{k=1}^{n} \varepsilon_{k} \cdot \frac{T}{(2 k+1) \pi} \cdot t .
\end{aligned}
$$

From Eq. (5), it is derived that

$V(T)=\sum_{k=1}^{n} \varepsilon_{k} \cdot \frac{2 T}{(2 k+1) \pi} \neq 0$; from Eq. (6), it is obtained $D(T)=\sum_{k=1}^{n} \varepsilon_{k} \cdot \frac{T^{2}}{(2 k+1) \pi} \neq 0$. Both velocity and displacement time histories do not satisfy the zero end conditions and present drift. On the other hand, if $V(t)$ and $D(t)$ are made to satisfy the zero end conditions, then they will not satisfy the zero initial conditions. Therefore, drift in velocity and displacement time histories from integrating acceleration time history cannot be avoided.

It is noted that, this study does not deny the contribution of other factors (such as noise) for the drift of displacement. As discussed in previous studies $[15,16]$, the distortions and errors from various sources (such as perturbations of the base of the seismic instrument in the form of rotations and tilts, and digitization errors) embedded in the recorded acceleration signal could contribute to the drift in displacement.

\subsection{Eigenfunction}

To resolve the over-determinacy associated with integration when determining velocity and displacement consistent with the known acceleration, Li et al. [5] proposed the eigenfunction.

In the study of Li et al. [5], the displacement eigenfunction is expressed as

$$
\begin{aligned}
& \varphi(t)=C_{1} \cos \lambda t+C_{2} \sin \lambda t+\exp \left(\frac{\sqrt{3} \lambda t}{2}\right) \\
& \left(C_{3} \cos \frac{\lambda t}{2}+C_{4} \sin \frac{\lambda t}{2}\right)+\exp \left(-\frac{\sqrt{3} \lambda t}{2}\right)\left(C_{5} \cos \frac{\lambda t}{2}+C_{6} \sin \frac{\lambda t}{2}\right) .
\end{aligned}
$$

The velocity eigenfunction $\dot{\varphi}(t)$ and the acceleration eigenfunction $\ddot{\varphi}(t)$ are written as,

$$
\begin{aligned}
& \dot{\varphi}(t) \frac{2}{\lambda}=-2 C_{1} \sin \lambda t+2 C_{2} \cos \lambda t \\
& +\exp \left(\frac{\sqrt{3} \lambda t}{2}\right)\left[C_{3}\left(\sqrt{3} \cos \frac{\lambda t}{2}-\sin \frac{\lambda t}{2}\right)+C_{4}\left(\cos \frac{\lambda t}{2}+\sqrt{3} \sin \frac{\lambda t}{2}\right)\right] \\
& -\exp \left(-\frac{\sqrt{3} \lambda t}{2}\right)\left[C_{5}\left(\sqrt{3} \cos \frac{\lambda t}{2}+\sin \frac{\lambda t}{2}\right)+C_{6}\left(\cos \frac{\lambda t}{2}-\sqrt{3} \sin \frac{\lambda t}{2}\right)\right], \\
& \ddot{\varphi}(t) \cdot \frac{2}{\lambda^{2}}=-2 C_{1} \cos \lambda t-2 C_{2} \sin \lambda t \\
& +\exp \left(\frac{\sqrt{3} \lambda t}{2}\right)\left[C_{3}\left(\cos \frac{\lambda t}{2}-\sqrt{3} \sin \frac{\lambda t}{2}\right)+C_{4}\left(\sqrt{3} \cos \frac{\lambda t}{2}+\sin \frac{\lambda t}{2}\right)\right] \\
& -\exp \left(-\frac{\sqrt{3} \lambda t}{2}\right)\left[C_{5}\left(\cos \frac{\lambda t}{2}-\sqrt{3} \sin \frac{\lambda t}{2}\right)+C_{6}\left(\sqrt{3} \cos \frac{\lambda t}{2}+\sin \frac{\lambda t}{2}\right) .\right.
\end{aligned}
$$

The coefficients $C_{1}, C_{2}, \ldots, C_{6}$ in Eqs. (7) to (9) are determined as follows,

$\left[\begin{array}{cccccc}1 & 0 & 1 & 0 & 1 & 0 \\ 0 & 2 & \sqrt{3} & 1 & -\sqrt{3} & 1 \\ -2 & 0 & 1 & \sqrt{3} & 1 & -\sqrt{3} \\ \cos v & \sin v & e^{\frac{\sqrt{3} v}{2}} \cos \frac{v}{2} & e^{\frac{\sqrt{3} v}{2}} \sin \frac{v}{2} & e^{-\frac{\sqrt{3} v}{2}} \cdot \cos \frac{v}{2} & e^{-\frac{\sqrt{3}}{2}} \sin \frac{v}{2} \\ -2 \sin v & 2 \cos v & e^{\frac{\sqrt{3} v}{2}} \cdot \mathrm{CS}^{-} & e^{\frac{\sqrt{3} v}{2}} \cdot \mathrm{SC}^{+} & -e^{-\frac{\sqrt{3} v}{2}} \cdot \mathrm{CS}^{+} & -e^{-\frac{\sqrt{3} v}{2}} \cdot \mathrm{SC}^{-} \\ -2 \cos v & -2 \sin v & -e^{\frac{\sqrt{3} v}{2}} \cdot \mathrm{CS}^{-} & e^{\frac{\sqrt{3} v}{2}} \cdot \mathrm{CS}^{+} & e^{-\frac{\sqrt{3} v}{2}} \cdot \mathrm{CS}^{+} & -e^{-\frac{\sqrt{3} v}{2}} \cdot \mathrm{CS}^{-}\end{array}\right]\left[\begin{array}{l}C_{1} \\ C_{2} \\ C_{3} \\ C_{4} \\ C_{5} \\ C_{6}\end{array}\right]=0,($

where

$$
\mathrm{CS}^{ \pm}=\sqrt{3} \cos \frac{v}{2} \pm \sin \frac{v}{2}, \quad \mathrm{SC}^{ \pm}=\sqrt{3} \sin \frac{v}{2} \pm \cos \frac{v}{2}, \quad v=\lambda T .
$$

For $C_{1}, C_{2}, \ldots, C_{6}$ to have nontrivial solutions, the determinant of the coefficient matrix should be 0 , yielding the eigenequation:

$8 \sin v-\sin 2 v-16 \sin \frac{v}{2} \times \cosh \frac{\sqrt{3} v}{2}+2 \sin v \times \cosh \sqrt{3} v=0$

The eigenequation (Eq. (11)) is a transcendental equation and has infinitely many roots or eigenvalues. It can be shown that there are two sets of roots:

1. The first set of roots are given exactly by $\sin (v / 2)=0: v=2 k \pi, k=1,2, \ldots$

2. The second set of roots are 9.4270555709, 15.7079533785, 21.9911486180, 28.2743338821, etc., which are given approximately by $\cos (v / 2)=0$ : $v=(2 k+1) \pi, k=1,2, \ldots$. It is impossible to find analytically exact solutions; but for $v>30$, the approximation is quite good with relative error smaller than $10^{-12}$.

Therefore, the eigenvalues can be written as $v_{n}=\lambda_{n} T=(n+1) \pi, \quad n=1,2, \ldots$, in which the results are exact when $n$ is odd and approximate when $n$ is even. 
For each eigenvalue $v_{n}$, the corresponding eigenvector $\boldsymbol{C}_{n}=\left\{C_{n 1}, C_{n 2}, \ldots, C_{n 6}\right\}^{T}, n=1,2, \ldots$, can be otbained from Eq. (10) and the eigenfunction $\varphi_{n}(t)$ is determined from Eq. (7).

\subsection{Determine velocity and displacement time histories} In this study, the eigenfunction method is proposed to determine displacement and velocity from acceleration data. For acceleration time history $A(t)$ with duration $T$ and sampled at $N$ discrete time instances $t_{i}=(i-1) \Delta t$, $i=1,2, \ldots, N$, where $\Delta t=T /(N-1)$, a procedure to determine $V(t)$ and $D(t)$ is proposed as follows:

1. If the acceleration shows near-zero values at both ends (i.e., the acceleration approximately satisfies the zero initial and end conditions), add zeros at both ends. The new zero-padded acceleration $\bar{A}(t)$ has the duration $\bar{T}$, and sampled at $\bar{N}$ discrete time instances; $\bar{N}-N$ denotes the total number of zero-padded portion at both ends.

2. Decompose zero-padded $\bar{A}(t)$ using a set of $\bar{N}$ eigenfunctions (the number of eigenfunctions is the same with the number of discrete time instances):

$$
\bar{A}(t)=\sum_{n=1}^{\bar{N}} a_{n} \ddot{\varphi}_{n}(t) .
$$

3. Multiply both sides of Eq. (12) by $\ddot{\varphi}_{m}(t), m=1,2, \ldots, \bar{N}$ and integrate is from 0 to $\bar{T}$ :

$$
\int_{0}^{\bar{T}} \bar{A}(t) \ddot{\varphi}_{m}(t)=\sum_{n=1}^{\bar{N}} a_{n} \int_{0}^{\bar{T}} \ddot{\varphi}_{n}(t) \ddot{\varphi}_{m}(t) .
$$

Let

$$
r_{m}=\int_{0}^{\bar{T}} \bar{A}(t) \ddot{\varphi}_{m}(t), \quad \Phi_{m n}=\int_{0}^{\bar{T}} \ddot{\varphi}_{m}(t) \ddot{\varphi}_{n}(t),
$$

then Eq. (13) becomes

$$
r_{m}=\sum_{n=1}^{\bar{N}} \Phi_{m n} a_{n}, \quad m=1,2, \ldots, \bar{N} .
$$

4. Solve the and determine the coefficients of expansion $a_{n}$, where $n=1,2, \ldots, \bar{N}$.

5. Determine velocity and displacement time histories using the coefficients of expansion $a_{n}$ :

$$
\bar{V}(t)=\sum_{n=1}^{\bar{N}} a_{n} \dot{\varphi}_{n}(t), \quad \bar{D}(t)=\sum_{n=1}^{\bar{N}} a_{n} \varphi_{n}(t) .
$$

6. Remove zero-padded portions of $\bar{V}(t)$ and $\bar{D}(t)$ at both ends, and obtain the velocity $V(t)$ and displacement $D(t)$ consistent with the original acceleration $A(t)$.
It is noted that, the velocity $V(t)$ and displacement $D(t)$ obtained through zero-padded $\bar{A}(t)$ can avoid the unrealistic value of zero at both ends of velocity $V(t)$ and displacement $D(t)$, which is enforced by the eigenfunction. Thus, velocity $V(t)$ and displacement $D(t)$ may show some minor values at both ends; this does not contradict the starting point of the proposed eigenfunction method because the zero-padded time history, including $\bar{A}(t), \bar{V}(t)$, and $\bar{D}(t)$, satisfy the zero initial and end conditions.

\section{Validation}

In Section 3, two examples are adopted to validate the eigenfunction method in this study to determine the displacement. The first example uses the recorded ground motion, and the second example uses one set of experiment data (including the measured acceleration and displacement time histories), which was provided by Dr. Claudia C. Marin-Artieda from the Howard University.

In the first example, the El Centro earthquake ground motions (E-W component, including consistent acceleration, velocity, and displacement time histories) from Pacific Earthquake Engineering Research Center (PEER) strong motion database are used. The velocity and displacement time histories determined by the eigenfunction method are shown in Fig. 1. It is seen that the velocity and displacement time histories determined by the eigenfunction method exactly match the velocity and displacement provided by the PEER strong motion database.

In the second example, the measured experiment data was recorded on an earthquake simulation testing program that was conducted in 2014 at the Structural Engineering and Earthquake Simulation Laboratory, at the State University of New York at Buffalo. K600 Krypton camera and LED sensors were used to capture the displacement time histories. The accuracy of the displacement time histories defined by the Krypton camera is $1: 5000 \pm 1 \mathrm{~mm}$ [17].

In the experiment, an input acceleration to simulate the $120 \%$ of the recorded roof acceleration at San Bernardino 5-story hospital, station CSMIP23634, channels 9 under the Landers earthquake (California Integrated Seismic Network, CISN) was applied to a seismic isolated system that support a simulated equipment [17]. The acceleration and displacement responses of the seismic isolated system were recorded by sensors.

Simple processing has been performed to these raw accelerations recorded by sensors. First, remove the mean of the raw acceleration. Next, apply the Butterworth filtering to remove the noise with frequencies lower than 
$0.1 \mathrm{~Hz}$. The eigenfunction method is then applied to the processed accelerations, and determine corresponding displacements. To evaluate accuracy of the eigenfunction method to determine the displacement time history, a parameter of relative error $e_{t}$ is defined as $\left(Y_{t}-C_{t}\right) / \mathrm{pgd}$, where $Y_{t}$ denotes the recorded displacement time history, $C_{t}$ denotes the computed displacement time history, and pgd denotes the maximum absolute value of $Y_{t}$. The Mean Squared Error (MSE) based on $e_{t}$ is defined as

$$
\operatorname{MSE}=\frac{1}{n} \sum_{t=1}^{n} e_{t}^{2},
$$

where $n$ denotes the total number of the discrete time history.
Fig. 2 and Fig. 3 show input time histories and response time histories of the seismic isolated system, respectively. Fig. 2(b) and Fig. 3(b) demonstrate that the displacement determined by the eigenfunction method. Values of MSE for the displacement time histories in Fig. 2(b) and Fig. 3(b) are 0.0221 and 0.0105 , respectively. The displacement determined by the eigenfunction method matches the measured displacement, although some minor difference exist, which may be due to the noise in the measured displacement.

To investigate the displacement calculated through baseline correction, double integration is firstly applied to the processed input acceleration, and corresponding displacement is determined, as seen in Fig. 4(a), in comparison
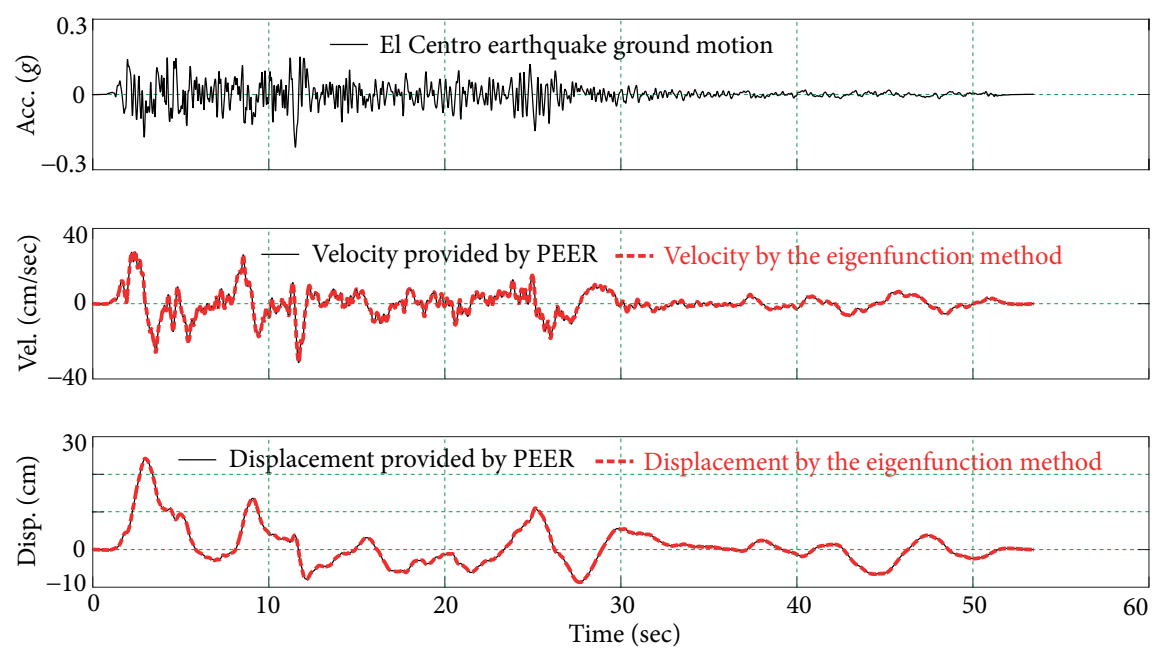

Fig. 1 Velocity and displacement time histories determined by the eigenfunction method
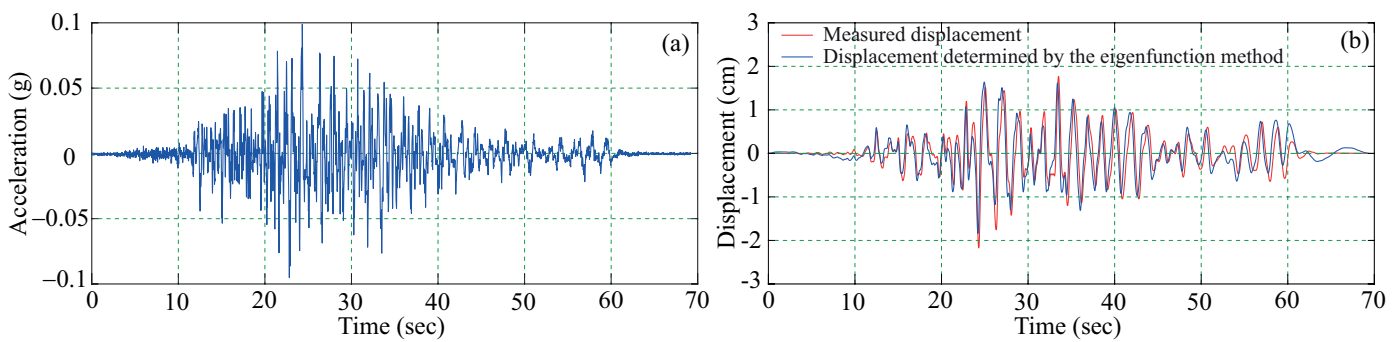

Fig. 2 Input time histories to the seismic isolated system: (a) acceleration; (b) displacement
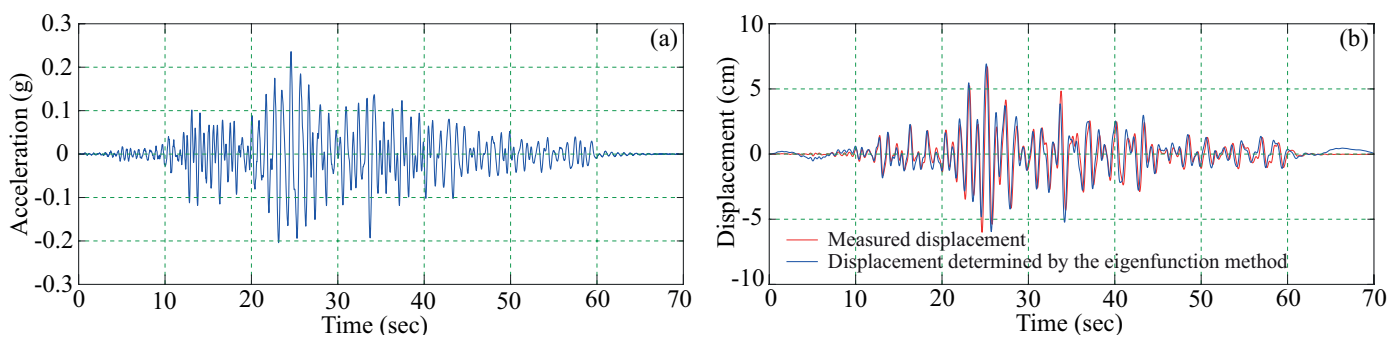

Fig. 3 Response time histories of the seismic isolated system: (a) acceleration; (b) displacement 
with the measured displacement. Fig. 4(a) shows that the displacement by integration presents some errors in comparison with the measured displacement. Particularly at points near the end of the time history, where the displacement by double integration remarkably deviates from the measured displacement and presents drift. The value of MSE is equal to 0.0326 . To remove the drift, baseline correction (including linear, quadratic, and cubic polynomial methods) is performed to the processed input acceleration using SeismoSignal software [18]. Then, the corresponding displacements are calculated by integration, as presented in Fig. 4(b) (Because the baseline correction using the cubic polynomial method yields almost the same result as compared with that of the quadratic polynomial method, the displacement from cubic polynomial method is not shown in this figure). Fig. 4(b) demonstrates that the baseline correction does not improve estimation of the displacement time history; the displacement time history still remarkably deviates from the measured displacement and present drifts near the end points. Values of MSE for the displacement time history using linear method and quadratic method are 0.0325 and 0.0357 , respectively. The drift is probably caused by the over-determinacy associated with the integration.

\section{Case studies}

\subsection{Case 1: seismic response analysis of a bridge}

In this case example, finite element method is used to analyze the seismic responses of a nine-span viaduct steel girder bridge subject to a set of tri-directional ground motions. A finite element model of the bridge is established by the SAP2000, as seen in Fig. 5.

The tri-directional ground motions recorded at the station "Beverly Hills-14145 Mulhol" during the 1994 Northridge Earthquake are used as seismic excitations to simulate dynamic responses of the bridge. The original tri-directional ground motions are scaled to the same peak ground acceleration, i.e., $0.15 \mathrm{~g}$. Plots of these scaling tri-directional ground motions are presented in Fig. 6(a). The horizontal-1, horizontal-2, and vertical components are respectively applied to the longitudinal, transverse, and vertical directions of the bridge in simulation.

Seismic responses (including relative acceleration, relative velocity, and relative displacement responses) of one selected point, i.e., joint 1 (see Fig. 5), are calculated by the SAP2000 and shown in Fig. 6(b) and Fig. 7. The relative velocity and displacement responses calculated by the SAP2000 are used as benchmark. Using relative acceleration responses calculated by the SAP2000, relative velocity and displacement responses are further determined by the eigenfunction method, in comparison with the benchmark, as presented in Fig. 6(b) and Fig. 7. They clearly show that the velocity and displacement responses determined by the eigenfunction method exactly match the benchmark. This example demonstrates that the eigenfunction method could determine accurate velocity and displacement responses of vibrating structures from simulated acceleration responses.

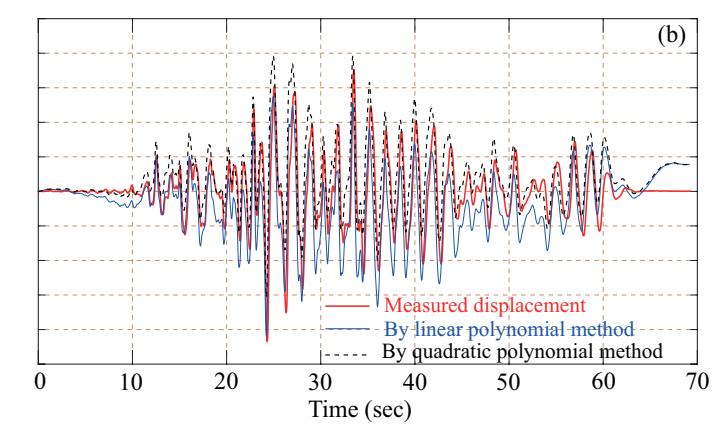

Fig. 4 Input time history: (a) measured displacement and displacement determined by integration; (b) measured displacement and displacement determined through baseline correction

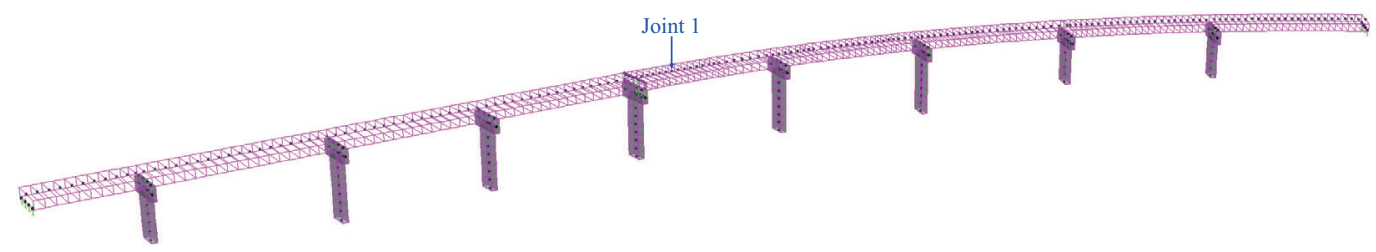

Fig. 5 Finite element model of the bridge in SAP2000 

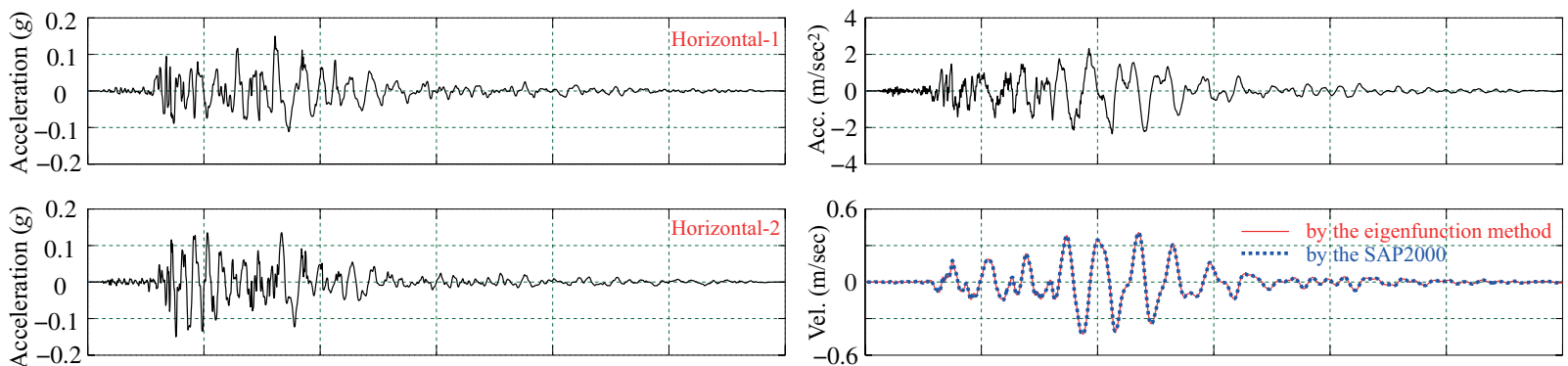

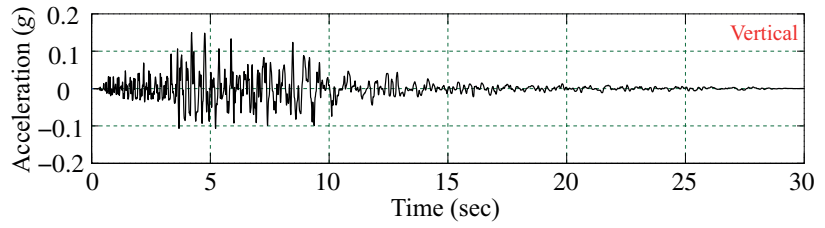

(a)

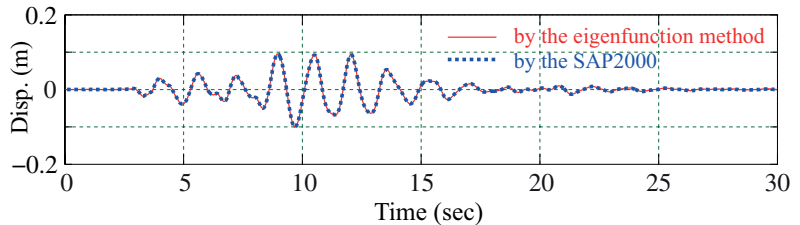

(b)

Fig. 6 Time history for (a) input motions for seismic response analysis; (b) response time histories in the longitudinal direction
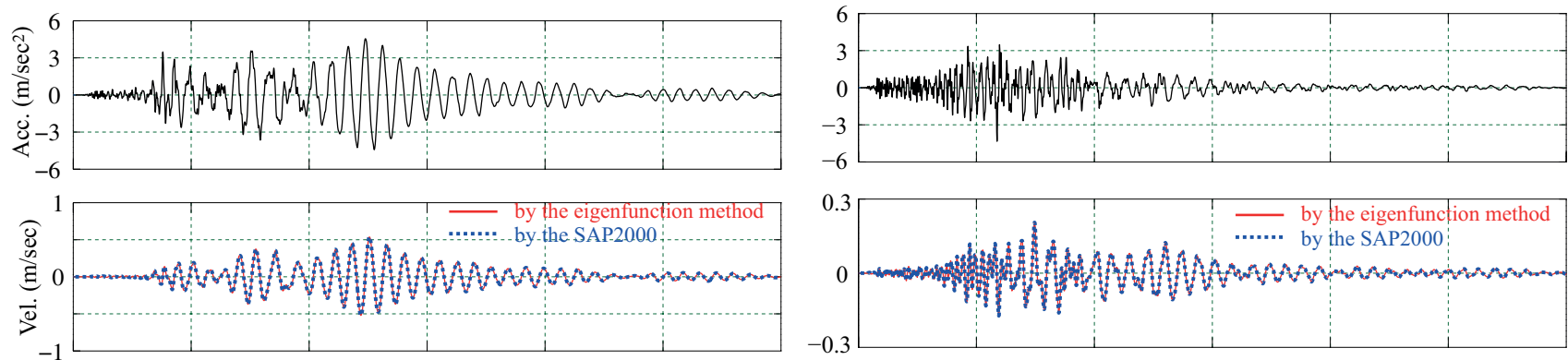

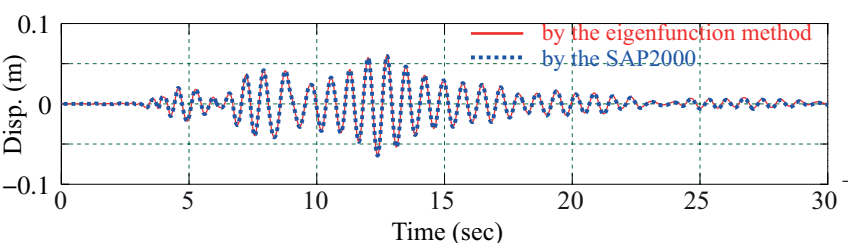

(a)

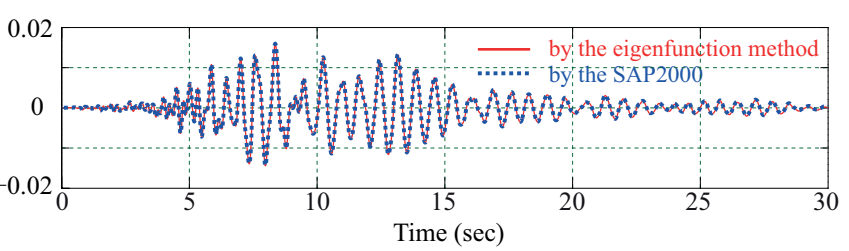

(b)

Fig. 7 Response time histories (a) in the transverse direction; (b) in the vertical direction

\subsection{Case 2: structural response measurement}

In this case example, based on sensor-measured accelerations of Painter Street Overpass (PSO), velocity and displacement are determined by the eigenfunction method. PSO is a two-span, prestressed concrete box-girder bridge. It has end diaphragm abutments and a two-column bent. The bridge was constructed in 1973 to cross over the fourlane US Highway 101 in Rio Dell, California. After construction of the bridge, it was instrumented with 20 strong motion sensors. The general dimension and parts of strong motion instrumentation are shown in Fig. 8.

Until now, accelerograms of 18 earthquakes have been recorded by these sensors. These recorded accelerograms are all available at Center for Engineering Strong Motion Data (CESMD), and have been processed and reviewed by California Geological Survey or United States Geological Survey. In this case example, accelerograms recorded during the Petrolia Earthquake in 1992 are used; one accelerogram recorded at ground near abutment of the bridge, and one accelerogram recorded at the bridge deck, are particularly selected. As seen in Fig. 8, Channel 17 measured accelerograms at ground near eastern abutment, and Channel 7 measured accelerograms at middle of the bridge deck.

The accelerograms recorded at the eastern abutment of the bridge and at the middle of bridge deck are respectively presented in Fig. 9 and Fig. 10, together with their corresponding velocity and displacement time histories obtained by direct numerical integration and by the eigenfunction method. Previous seismic investigation [20] indicated that the Petrolia Earthquake in 1992 did not cause any permanent deformation at the bridge or the ground. Thus, the displacement of these accelerograms should be close to 0 at the end of the shaking. 


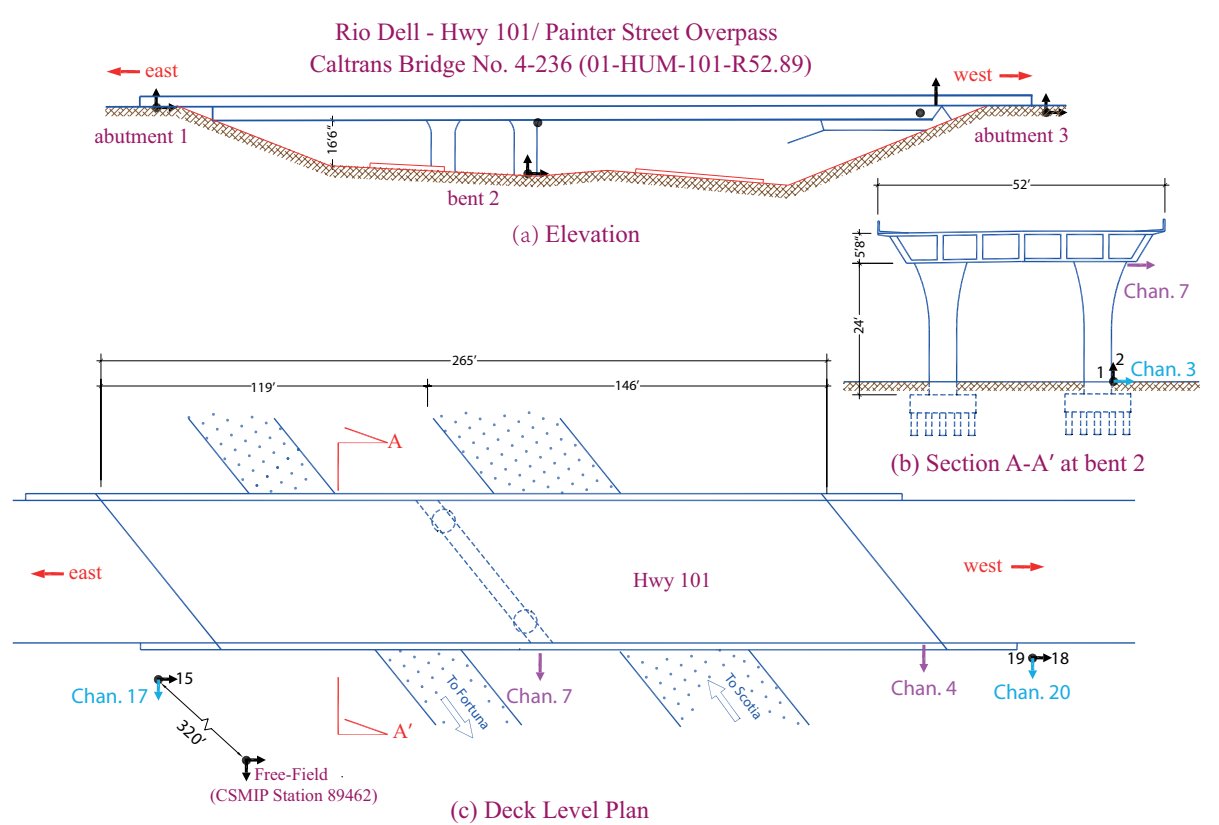

Fig. 8 Distribution of parts of strong motion instrumentation at PSO (figure modified based on drawing at CESMD [19])
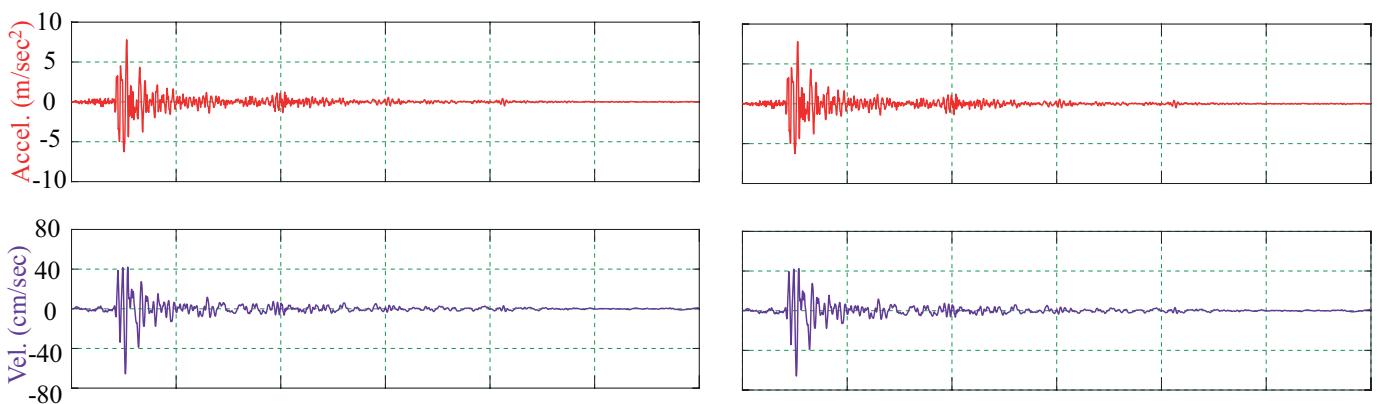

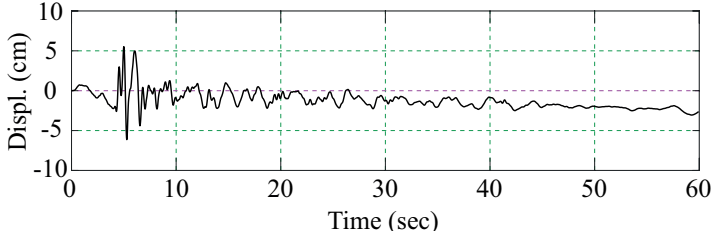

(a)

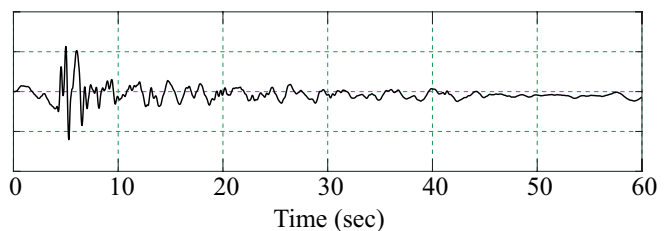

(b)

Fig. 9 Accelerogram at the eastern abutment and its velocity and displacement (a) by direct numerical integration; (b) by the eigenfunction method

Fig. 9 demonstrates that displacement time history obtained by the numerical integration presents a slight drift, with a value of $-2.62 \mathrm{~cm}$ at the terminal. The displacement time history determined by the eigenfunction method presents a value of $-0.67 \mathrm{~cm}$ at the terminal, which is closer to 0 , in comparison with the peak value of displacement time history, i.e., $6.03 \mathrm{~cm}$. Fig. 10 indicates that, the displacement time history obtained by the numerical integration shows remarkable drift at the terminal, with a value of $-5.28 \mathrm{~cm}$. The displacement time history determined by the eigenfunction method presents a value of $-0.66 \mathrm{~cm}$ at the terminal, which is also close to 0 , in comparison with the peak value of the displacement time history, i.e., $6.94 \mathrm{~cm}$. The remarkable drift in the displacement time history determined by numerical integration contradicts the fact of no permanent deformation with the bridge deck after the earthquake [20].

It is noted that, because the displacement time histories in Fig. 9 and Fig. 10 were obtained from the eigenfunction method through zero-padded $\bar{A}(t)$ avoid the unrealistic zero value at both ends of displacement. The displacement time histories determined by the eigenfunction method show some minor values at both ends; this does not contradict the starting point of the proposed eigenfunction method because the zero-padded time history exactly satisfy the zero initial and end conditions. 

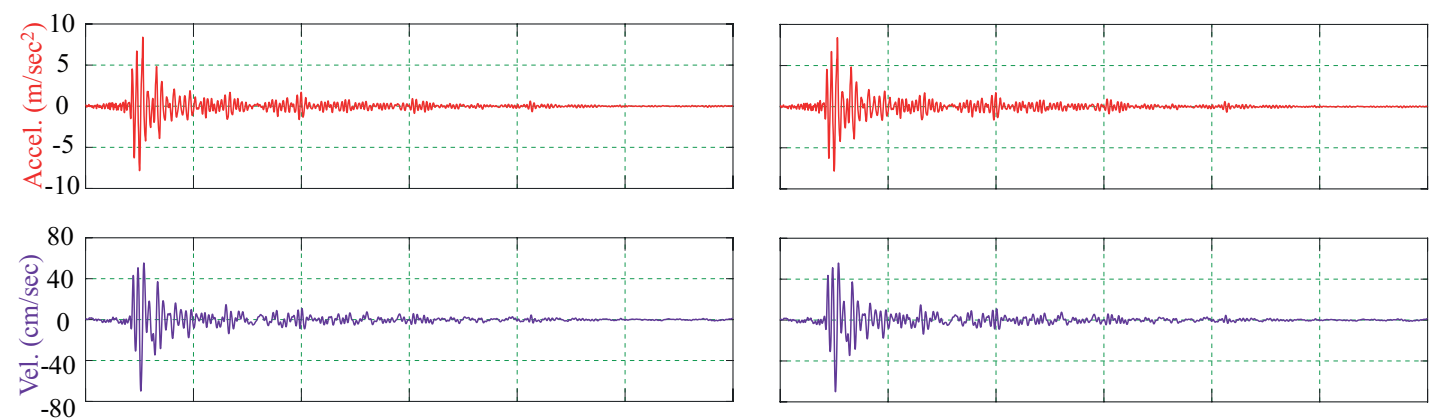

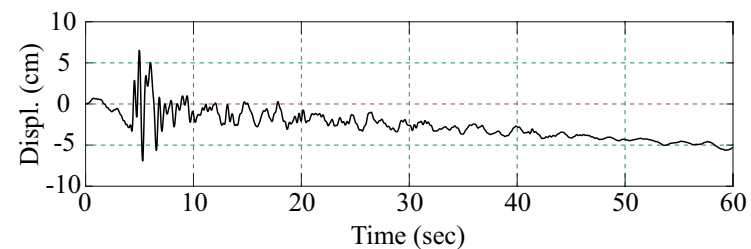

(a)

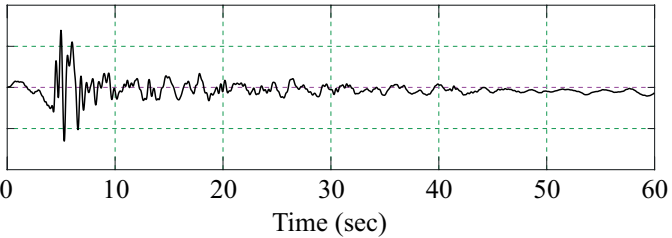

(b)

Fig. 10 Accelerogram at middle bridge deck and its velocity and displacement (a) by direct numerical integration; (b) by the eigenfunction method

This example demonstrates that displacement time histories obtained by the numerical integration may cause misleading judgement with respect to deformation of the bridge subjected to vibration. It may also provide misleading information with respect to integrity and serviceability of a structure during structural health assessment. Using the eigenfunction method, realistic displacement time histories could be determined, and dependent structural information could be derived accurately.

\section{Conclusions}

In this study, a new method was proposed to determine velocity and displacement of vibrating structures from acceleration data. A major advantage of the proposed method is that computed velocity and displacement do not have any unrealistic drift. In addition, because the eigenfunction method is derived analytically, the velocity and displacement obtained by this method do not suffer from numerical errors and the problem of over-determinacy associated with the numerical integration method.

This paper also presented one recorded ground motion and one set of experiment data to validate the eigenfunction method. In addition, two case studies also demonstrated the capabilities of the eigenfunction method to determine velocity and displacement. Efficiency of the

\section{References}

[1] Şafak, E. "Detection and Identification of Soil-Structure Interaction in Buildings from Vibration Recordings", Journal of Structural Engineering, 121(5), pp. 899-906, 1995. https://doi.org/10.1061/(ASCE)0733-9445(1995)121:5(899) eigenfunction method to derive displacement from acceleration data was illustrated in these two case studies. The displacement determined by the eigenfunction method could be further used to solve engineering problems such as inter-story drift ratio of buildings, soil-structure interaction analysis etc. It is necessary to mention that the eigenfunction method cannot be applied to determine permanent ground displacement.

\section{Acknowledgements}

The research for this study was supported, in part, by the Fundamental Research Funds for the Central Universities (Grant Nos. YJ201824 and 19XJ0077), the National Natural Science Foundation of China (Grant No. U1710111) and International Collaboration Program of Science and Technology Commission of Sichuan Province (Grant No. 18GJHZ0111), the Natural Sciences and Engineering Research Council of Canada (NSERC), and University Network of Excellence in Nuclear Engineering (UNENE). We are also very thankful to Dr. Claudia C. Marin-Artieda from the Howard University, for providing us with the measured experiment data in the earthquake simulation testing program that was conducted in 2014 at the Structural Engineering and Earthquake Simulation Laboratory, at the State University of New York at Buffalo.

[2] Yang, J., Li, J. B., Lin, G. "A simple approach to integration of acceleration data for dynamic soil-structure interaction analysis", Soil Dynamics and Earthquake Engineering, 26(8), pp. 725-734, 2006. https://doi.org/10.1016/j.soildyn.2005.12.011 
[3] Sullivan, T. J. "Direct displacement-based seismic design of steel eccentrically braced frame structures", Bulletin of Earthquake Engineering, 11(6), pp. 2197-2231, 2013.

https://doi.org/10.1007/s10518-013-9486-8

[4] Skolnik, D. A., Wallace, J. W. "Critical Assessment of Interstory Drift Measurements", Journal of Structural Engineering, 136(12), pp. 1574-1584, 2010.

https://doi.org/10.1061/(ASCE)ST.1943-541X.0000255

[5] Li, B., Ly, B. L., Xie, W. C., Pandey, M. D. "Generating SpectrumCompatible Time Histories Using Eigenfunctions", Bulletin of the Seismological Society of America, 107(3), pp. 1512-1525, 2017. https://doi.org/10.1785/0120160206

[6] Boore, D. M. "Effect of Baseline Corrections on Displacements and Response Spectra for Several Recordings of the 1999 ChiChi, Taiwan, Earthquake", Bulletin of the Seismological Society of America, 91(5), pp. 1199-1211, 2001.

https://doi.org/10.1785/0120000703

[7] Iwan, W. D., Moser, M. A., Peng, C. Y. "Some observations on strong-motion earthquake measurement using a digital accelerograph", Bulletin of the Seismological Society of America, 75(5), pp. 1225-1246, 1985. [online] Available at: http://bssa.geoscienceworld.org/content/75/5/1225.abstract [Accessed: 12 October 2019]

[8] Converse, A., Brady, A. G. "BAP: Basic Strong-Motion Accelerogram Processing Software, Version 1.0", U.S. Department of the Interior, U.S. Geological Survey, Virginia, USA, Rep. 92-296B, 1992.

https://doi.org/10.3133/ofr92296B

[9] Boore, D. M., Azari Sisi, A., Akkar, S. "Using Pad-Stripped Acausally Filtered Strong-Motion Data", Bulletin of the Seismological Society of America, 102(2), pp. 751-760, 2012. https://doi.org/10.1785/0120110222

[10] Iwan, W. D., Chen, X. "Important near-field ground motion data from the landers earthquake", In: 1994 10th European Conference on Earthquake Engineering, Vienna, Austria, 1994, pp. 229-234.

[11] Zhou, Y., Zhang, W., Yu, H. "Analysis of long-period error for accelerograms recorded by digital seismographs", Earthquake Engineering and Engineering Vibration, 17(2), pp. 1-9, 1997.

https://doi.org/10.13197/j.eeev.1997.02.001
[12] Whitney, R. "Ground motion processing and observations for the near-field accelerograms from the 2015 Gorkha, Nepal earthquake", Soil Dynamics and Earthquake Engineering, 107, pp. 250-263, 2018. https://doi.org/10.1016/j.soildyn.2018.01.032

[13] Yang, L., Xie, W. C., Xu, W., Ly, B. L. "Generating Drift-Free, Consistent, and Perfectly Spectrum-Compatible Time Histories", Bulletin of the Seismological Society of America, 109(5), pp. 16741690, 2019. https://doi.org/10.1785/0120190005

[14] Li, B., Xie, W. C., Pandey, M. D. "Generate tri-directional spectra-compatible time histories using HHT method", Nuclear Engineering and Design, 308, pp. 73-85, 2016. https://doi.org/10.1016/j.nucengdes.2016.08.009

[15] Boore, D. M., Bommer, J. J. "Processing of strong-motion accelerograms: needs, options and consequences", Soil Dynamics and Earthquake Engineering, 25(2), pp. 93-115, 2005. https://doi.org/10.1016/j.soildyn.2004.10.007

[16] Graizer, V. M. "Effect of tilt on strong motion data processing", Soil Dynamics and Earthquake Engineering, 25(3), pp. 197-204, 2005. https://doi.org/10.1016/j.soildyn.2004.10.008

[17] Tezcan, J., Marin-Artieda, C. C. "Least-Square-Support-VectorMachine-based approach to obtain displacement from measured acceleration", Advances in Engineering Software, 115, pp. 357-362, 2018.

https://doi.org/10.1016/j.advengsoft.2017.10.011

[18] Seismosoft "SeismoSignal 2016-A computer program for signal processing of strong-motion data", Seismosoft Ltd, 2016. [online] Available at: http://www.seismosoft.com [Accessed: 12 October 2019]

[19] CESMD "Center for Engineering Strong Motion Data Internet Data Reports", [pdf] Center for Engineering Strong Motion Data, Available at: http://www.strongmotioncenter.org/NCESMD/photos/CGS/11layouts/1189324.pdf [Accessed: 12 October 2019]

[20] Ventura, C. E., Brincker, R., Andersen, P. "Dynamic Properties of the Painter Street Overpass at Different Levels of Vibration", In: 2005 6th International Conference on Structural Dynamics (EURODYN), Paris, France, 2005, pp. 167-172. [online] Available at: https://portal.findresearcher.sdu.dk/en/publications/ dynamic-properties-of-the-painter-street-overpass-at-different-le [Accessed: 12 October 2019] 